\title{
Satisfacción laboral en la gestión administrativa
}

\author{
Janina Saavedra Meléndez \\ psicologasaavedra@hotmail.com
}

José Manuel Delgado Bardales

jmdelgadob@ucvvirtual.edu.pe

Universidad César Vallejo

\section{RESUMEN}

El estudio tuvo como objetivo caracterizar la satisfacción laboral en la gestión administrativa. La investigación fue básica de diseño no experimental descriptiva con revisión sistemática. Se realizó como procedimiento de recolección de datos una tabla de registro de datos y la fuente fue la revisión bibliográfica de artículos científicos nacionales e internacionales, que tuvieron como tema la gestión administrativa y la satisfacción laboral, se escogió 11 artículos más relevantes. En conclusión, La satisfacción laboral en la gestión administrativa es como se siente el trabajador en su ambiente de trabajo, los colaboradores de estas instituciones no están del todo satisfechos, por las bajas remuneraciones, los ambientes de trabajo inadecuados y el deficiente liderazgo. La gestión administrativa necesita de estrategias que permita direccionar la organización, pues eso ayuda al cumplimiento de metas, la dirección, organización, control y planificación deben ser utilizados adecuadamente. La satisfacción laboral es direccionada por el área de los recursos humanos quien toma en cuenta a los colaboradores como el centro de toda organización para reconocer y motivar el desempeño laboral.

Palabras clave: satisfacción laboral, gestión administrativa, colaboradores. 


\title{
Job satisfaction in administrative management
}

\begin{abstract}
The study aimed to characterize job satisfaction in administrative management. The research was of a basic descriptive non-experimental design with a systematic review. As a data collection procedure, a data record table was carried out and the source was the bibliographic review of national and international scientific articles, which had administrative management and job satisfaction as a theme, 11 most relevant articles were chosen. In conclusion, Job satisfaction in administrative management is how the worker feels in their work environment, the collaborators of these institutions are not entirely satisfied, due to the low wages, inadequate work environments and poor leadership. Administrative management needs strategies that allow directing the organization, as this helps to achieve goals, direction, organization, control and planning must be used properly. Job satisfaction is directed by the human resources area, which takes into account employees as the center of any organization to recognize and motivate job performance.
\end{abstract}

Keywords: job satisfaction, administrative management, collaborators

Artículo recibido: 03 nov. 2020 Aceptado para publicación: 07 dic. 2020 Correspondencia psicologasaavedra@hotmail.com Conflictos de Interés: Ninguna que declarar 


\section{INTRODUCCIÓN}

Las diversas instituciones públicas y privadas tienen muchas formas de gestión administrativa, desde la gestión de los recursos financieros y los recursos humanos, estas instituciones invierten muchas horas y recursos en la planificación de sus actividades para llegar a los objetivos que se proponen, donde los colaboradores cumplen un papel importante para el cumplimiento de los mismo. La gestión administrativa es significativa, ya que ayuda en la rutina de trabajo, las estrategias, la gestión del tiempo y planificación de una organización; sumado a esta planificación están las personas que laboran quienes deben sentirse satisfechos con su lo que realizan. El éxito de las organizaciones modernas radica en la habilidad de integrar a los colaboradores en los proyectos y planificación, de tal forma que los ellos empiecen a ver los objetivos de la organización como suyos. Considerando a las personas como la base para lograr la competitividad, es importante contar con el personal idóneo y motivado (Sánchez y García, 2017, p.1).

Asimismo, las instituciones tienen el deseo de realizar una adecuada gestión administrativa, con políticas claras dirigidas a reducir las tasas de insatisfacción laboral y así mejorar el rendimiento de los colaboradores, además busca tener a las personas contentas en el ambiente laboral, pues no podemos hablar de salud, sin considerar la salud mental de las personas que enfrentan diferentes problemas en la vida diaria, más aún cuando no se sienten satisfechos en su área de trabajo, lo cual puede generar bajo nivel de rendimiento. La satisfacción laboral tiene que ver con, cómo las personas perciben su trabajo, las funciones que realiza y el reconocimiento que recibe, así como a las actitudes que presenta frente al mismo, las opiniones que puede tener del trabajo y de su funcionamiento.

Sin embargo, en los estudios de Sánchez, Fuentes y Artacho, (2018) en los rankings internacionales de satisfacción laboral encontraron algunos resultados que muestran la realidad frente a este problema: en España la mayoría de los trabajadores tiene un nivel medio de satisfacción laboral, las principales causas son el bajo salario y el mal horario de trabajo seguido de la inestabilidad laboral. En México sobre el valor percibido del trabajador respecto a la satisfacción laboral, concluyeron que el 75\% de los encuestados les agrada las tareas que hacen en su trabajo, en los indicadores evaluados las medias son altas, por lo que los colaboradores notan que la empresa los toma en cuenta, así como que su 
empleo es enriquecedor e importante para el logro de objetivos (p.60-61). Asimismo, en Colombia, Sánchez y García (2017), encontraron que la satisfacción laboral de los trabajadores encuestados en ese país no es muy alta, debido a que se presentan algunas disconformidades respecto a los salarios y oportunidades de desarrollo profesional dentro de las empresas.

También, en el Perú se desarrolló un estudio de satisfacción laboral a través de una encuesta, participaron trabajadores de diversas empresas; se encontró que el 76\% de los peruanos no son felices en su trabajo, el $22 \%$ manifiesta que es porque no está en un trabajo desafiante, otro $20 \%$ dice que hay un mal clima laboral, el $19 \%$ de los encuestados refieren que le falta liderazgo a su jefe, el $12 \%$ dice que no está contento con su salario, el $10 \%$ opina que la empresa no reconoce sus logros y que no es libre de aportar con nuevas ideas, el 7\% indica que no le dan oportunidades de ascender y crecer profesionalmente. Así mismo, existe otro $24 \%$ que sí se siente contento con su trabajo; el $93 \%$ indica que su trabajo es fundamental para la organización (Diario Gestión, 2016).

Por otro lado, Córdova y Arévalo, (2018) realizaron una encuesta en la ciudad de Tarapoto, donde encontraron que los trabajadores participantes de la encuesta manifiestan que la gestión administrativa de su empresa se encuentra en nivel regular y en la satisfacción laboral se sienten satisfechos a un nivel regular, mostrándose que están relacionadas de manera considerable, a mayor gestión administrativa mayor satisfacción laboral. Cuando los trabajadores no se encuentran satisfechos, muestran actividades rutinarias, hacen el trabajo sólo por cumplir, les cuesta innovar en estrategias para lograr los objetivos comunes (p.36).

Por todo lo descrito los trabajadores manifiestan una deficiente satisfacción laboral, relacionado a la gestión administrativa, que es llevada de manera inadecuada, centrándose en cumplir solo los objetivos del trabajo, sin pensar en el personal que hace el trabajo diario, lo cual limita muchas veces el desarrollo profesional de los mismo, que se ve reflejado en la insatisfacción y el desempeño laboral. La importancia de la gestión administrativa radica, en la planificación, organización, dirección y control eficaz del trabajo a realizar.

Entonces se planteó el siguiente problema: ¿Cuáles son las características de la satisfacción laboral en la gestión administrativa? Asimismo, el objetivo fue caracterizar la satisfacción laboral en la gestión administrativa.

\section{ESTRATEGIAS METODOLÓGICAS O MATERIALES Y MÉTODOS}


La investigación fue básica de diseño no experimental descriptiva con revisión sistemática, las revisiones sistemáticas son síntesis estructuradas de la información orientada a una problemática de estudio. Son varios artículos y fuentes de información, que dan evidencias de alto nivel, es describir los procesos que siguieron los investigadores respecto a la recolección, selección, evaluación crítica de su investigación respecto a las variables de estudio (Moreno, Muñoz, Cuellas, Domancic, y Villanueva, 2018, p.184). Fue básica, no experimental, porque no va a aplicar ningún programa durante el estudio, no se manipulan las variables y descriptiva porque dio a conocer las características de la gestión administrativa y los niveles de satisfacción laboral. Por lo tanto, se revisó información científica, que mostró las características de la gestión administrativa y con ellos elevar los niveles de satisfacción laboral.

Se realizó como procedimiento de recolección de datos una tabla de registro de datos y la fuente fue la revisión bibliográfica de artículos científicos nacionales e internacionales, que tuvieron como tema la gestión administrativa y la satisfacción laboral, se escogió 11 artículos más relevantes que muestren datos relacionados al objetivo. La recolección de datos consiste en elaborar un plan detallado de los procedimientos que se van a utilizar para reunir los datos necesarios para la investigación, es decir tomar en cuenta las fuentes donde se obtendrán los datos, el medio y método; así mismo, este plan se nutre de las variables, la muestra, definiciones operacionales y recursos disponibles. (Hernández, Fernández y Baptista, 2010, p.198).

La técnica de análisis se desarrolló a través de la revisión sistemática, analizando cada uno de los artículos escogidos, para comparar los resultados y conclusiones de las investigaciones, tanto nacionales como internacionales. Además, se realizó un análisis crítico de cada artículo, para identificar la calidad y excelencia de la información que muestra para utilizarlo en el artículo científico.

\section{RESULTADOS Y DISCUSIÓN}

Los estudios revisados sobre la satisfacción laboral en la gestión administrativas son los siguientes: Face, M. y Herrera, S. (2017). La gestión administrativa y su vinculación en la satisfacción laboral, estudio realizado en México, publicada por la Revista Electrónica sobre Tecnología, Educación y Sociedad, 2017, 4, 7. Fue un estudio descriptivo no experimental, tuvo como muestra a 53 docentes de la Universidad Autónoma del Carmen, utilizó una escala de actitudes, basado en el código de ética en investigación. Los resultados 
fueron, los docentes encuestados se consideran satisfechos laboralmente, así mismo también se encuentran satisfechos con respecto a los factores relacionados con la gestión administrativa. Por otro lado, el factor que provoca mayor insatisfacción laboral es el sistema administrativo y las condiciones físicas de trabajo. Concluyen que, es buena la percepción sobre la gestión administrativa e influye de manera positiva en la satisfacción laboral. Existen factores externos como los horarios y la carga académica que ayudan a evitar, así mismo, hay factores internos del trabajo mismo, el desarrollo personal y la gobernabilidad que producen satisfacción, si estos factores son excelentes, ayudan a aumentar los niveles de excelencia, como lo dice la teoría bifactorial de Herzberg.

También se consideró a Pacheco, R. Robles, C. y Ospino, A. (2018). Realizaron una investigación sobre la Gestión Administrativa en las Instituciones Educativas en las Zonas Rurales de Colombia, y fue publicada en la revista electrónica Información Tecnológica, 2018, Vol. 29, 5, p.259-266. El tipo de estudio fue descriptivo de campo transversal, tuvo como participantes a 25 instituciones rurales de del distrito de Santa Marta, Colombia, considerando como instrumento un cuestionario tipo Likert donde se preguntaba aspectos de la gestión administrativa. Concluyeron que, la gestión administrativa existe moderada presencia del uso de técnicas de investigación operacional, también existe dos enfoques el clásico y el operacional, uno es el espacio para ver el rol del trabajador y asume que el trabajo diario es una evidencia de la entrega del personal a sus labores, el otro enfoque muestra al PEI como principal elemento de calidad; que se caracteriza por un estilo de dirección participativo, el cual ayuda a la toma de decisiones con el personal. La gestión administrativa está centrada en las malas decisiones de los rectores quienes administran el dinero girado por el estado para cada año fiscal, pues muchos de ellos utilizan el juicio personal para priorizar el gasto. También, no hay gestión de reuniones para la autoevaluación del avance del plan de mejoramiento institucional, asimismo, hacen un uso moderado de las técnicas de investigación operacional aplicadas a la gestión administrativa de instituciones educativas, por otro lado, la toma de decisiones es informal.

Asimismo, Caján, J. (2017). Desarrolló un modelo de gestión pedagógica utilizando el liderazgo transformacional para asegurar la satisfacción laboral de los docentes del ISEP “Sagrado Corazón de Jesús". Investigación realizada en Perú y publicada en UCV-HACER Revista de investigación y cultura, 2016, Vol. 5, 2. Se aplicó un cuestionario de Satisfacción Laboral a 26 docentes del ISEP Sagrado Corazón, es una investigación descriptiva 
propositiva. Concluyó que, los docentes muestran rechazo hacia la gestión institucional actual, lo que hace que tenga un nivel de satisfacción laboral bajo. Según la comunicación y coordinación, los docentes no están de acuerdo en la manera como se realiza en la institución educativa. En las condiciones ambientales e infraestructura consideran que no hay requerimiento que ayude en el trabajo diario del docente y el personal administrativo. Por otro lado, los docentes sienten que la motivación y reconocimiento, no es adecuada, y no se sienten motivados respecto a los horarios y salario. Por lo que, el autor de la investigación diseñó un modelo de gestión pedagógica enfocado en el liderazgo transformacional logrando garantizar la satisfacción laboral en sus dimensiones docencia, investigación, extensión y liderazgo académico. Con ello el docente fortalecerá su liderazgo, investigación y permanente capacitación, para llegar poco a poco a un nivel óptimo de satisfacción laboral.

Además, Olivares, J. et al. (2016). Investigó sobre la satisfacción laboral de docentes universitarios del Departamento Académico de Clínica Estomatológica. Perú. Fue publicada por la Revista Estomatológica Herediana, 2016, Vol. 16, 1, pp. 21-25. Es de tipo descriptivo, La población de estudio estuvo conformada por 51 docentes de la Facultad de Estomatología Roberto Beltrán Neira de la Universidad Peruana Cayetano Heredia tomando como muestra de estudio a 36 docentes. El instrumento utilizado fue una encuesta que medía de la satisfacción laboral, con una escala de Likert, los factores fueron: institución, remuneración, tensión laboral y condiciones laborales. Los resultados mostraron que el factor satisfacción de los docentes fue bueno. En el factor de remuneración obtuvo un puntaje de regular, en el factor de tensión laboral la opinión es regular, el factor según su condición laboral es regular. Concluyeron que, el nivel de satisfacción laboral global del personal docente fue regular, en cuanto a los factores tensión, laboral, remuneración, condición laboral fue regular y del factor satisfacción laboral por la institución fue buena.

También se revisó al estudio de Torres, O. Vallejos, A. y Burbano, J. (2019). Realizó un estudio en la ciudad de Ibarra sobre las buenas prácticas de gestión administrativa en empresas. Es un estudio descriptivo, participaron de 37 hoteles y 40 restaurantes, se encuestó a los trabajadores. Concluyen que, las acciones que implementan en su mayoría son aspectos positivos que les permite potenciar el uso de los materiales para el cumplimiento de las metas propuestas por la empresa. Las prácticas de los diferentes grupos 
de interés, permite a las empresas realizar procesos de gestión más eficientes. Las buenas prácticas se fundamentan y desarrollan en las personas, por tal motivo siempre en instancia se debe buscar el bienestar de los colaboradores, ya que, si ellos se encuentran comprometidos y a gusto con la empresa, será más sencillo y eficaz la implementación de las buenas prácticas de gestión empresarial, y, por ende, los resultados serán más evidentes. Por otro lado, Montes de Oca, J. y Pulla, C. (2019). La gestión administrativa y su influencia en la productividad de las microempresas ecuatorianas. Caso de estudio taller Dipromax de la ciudad de Santo Domingo. Estudio de República Dominicana, fue mostrada a la comunidad científica a través de la Revista Dilemas Contemporáneos: educación, Política y Valores, 2019, Vol. 7, 1. Se presentó una encuesta cerrada a 12 trabajadores y encuesta abierta a1 gerente de la empresa Dipromax. Encontraron que, no hay un adecuado plan de gestión, no hay visión, misión, no existe objetivos en conjunto con los colaboradores; así mismo, no existe capacitación para los colaboradores, no existe implementos de seguridad, lo que genera insatisfacción laboral y accidentes de trabajo. Por otro lado el ambiente físico es desordenado, con falta de espacio para realizar las labores, lo que genera desmotivación en los colaboradores; ya que, existe desorden y por ende la falta de espacios para realizar sus labores a diario. Concluyen que, la problemática encontrada se desarrolló una propuesta, buscando la solución al problema en el taller "Dipromax". El modelo de gestión administrativa fue estructurado considerando el análisis externo e interno, misión, visión, objetivos, estrategias, programas y presupuesto.

Asimismo, Bendezú, K. (2020), sobre gestión del talento humano y la satisfacción laboral de los colaboradores de una institución educativa Privada. Fue publicada en la revista peruana, Revista Investigación Valdizana, 2020, Vol. 14, 1, pp. 22-28. Estudio descriptivo correlacional, se aplicó la Escala de satisfacción laboral SL/SPC de la autora Sonia Palma a 18 docentes y 06 administrativos. Encontró que la gestión del talento humano se relaciona significativamente con la satisfacción laboral de los trabajadores, a nivel de correlación fuerte. Asimismo, a través de las dimensiones del comportamiento organizacional, comunicación, y compensación laboral, en la primera variable y las condiciones físicas y/o materiales, beneficios laborales y/o remunerativos, políticas administrativas, relaciones interpersonales, desarrollo personal, desempeño de tareas, relación con la autoridad para la segunda variable, se pudo conocer la opinión de los diferentes ambientes de trabajo. 
Además, Omar, A., Salessi, S. y Urteaga, F. (2017). Impact of management practices on job satisfaction. Artículo publicado en la RAM. Revista de Administração Mackenzie, 2017, Vol. 18, 5, pp. 92 - 115. Fue un estudio cuantitativo y transversal, asociativa explicativa, se utilizó la técnica de la encuesta y el instrumento Escala HRMP de 25 ítems, que media las dimensiones trabajo genérico y justicia organizacional. Concluyeron que, existía un alto nivel de satisfacción en el trabajo. Las personas están orientadas a los empleados y a los sistemas abiertos. Percepciones de justicia median parcialmente las relaciones entre el trabajador y su satisfacción.

También, Montoya, P., Bello, N., Bermúdez, N., Burgos, F., Fuentealba, M. y Padilla, A. (2017). Satisfacción laboral y su relación con el clima organizacional en colaboradores de una Universidad Estatal Chilena. El estudio fue publicado en la Revista Ciencia y trabajo, 2017, vol. 19, núm. 58, pp. 7 - 13. Estudio cuantitativo correlacional, se utilizó un cuestionario de Satisfacción Laboral S20/23 para la variable cima organizacional y un cuestionario sociodemográfico y laboral, fueron 166 trabajadores que participaron en el estudio. Encontraron que, la percepción de un clima organizacional alto se asocia a un mayor nivel de satisfacción laboral de docentes y administrativos. Se aprecia una adecuada satisfacción laboral en los trabajadores más antiguos.

Asimismo, Rodríguez, A., Gómez, P. y De Dios, R. (2017). Estudio de la satisfacción laboral en los equipos de atención primaria en un área sanitaria de Asturias. La investigación fue dada a conocer en la Revista electrónica trimestral de enfermería: Enfermería Global, 2017, Vol. 16 núm. 3, p. 369-383. Tipo de estudio fue descriptivo transversal, aplicaron el Cuestionario validado Font-Roja AP y un cuestionario sociodemográfico y laboral. Concluyeron que, existe una adecuada satisfacción laboral. Sin embargo, la percepción hacia el sueldo, promoción profesional y tensión en el trabajo es bajo.

Por último, Chiang, M. y San Martin, N. (2015). Análisis de la satisfacción y el desempeño laboral en los funcionarios de la Municipalidad de Talcahuano. El artículo fue publicado en la Revista Ciencia y trabajo, 2015, Vol. 17 núm. 54, pp. 159-165. Fue un estudio no experimental, de diseño transversal, tipo descriptivo y correlacional, donde participaron 259 funcionarios de la Municipalidad de Tulcahuano, se usó la técnica de la encuesta y aplicaron el cuestionario de desempeño laboral y cuestionario de satisfacción laboral. Concluye que, existe satisfacción laboral alta. Cuando se comparan ambos géneros 
coinciden en tener una mayor satisfacción en la relación que mantienen con sus superiores, el reconocimiento que se les entrega, y por trabajar dentro de ese departamento, dirección o institución. Por otro lado, presentan una baja satisfacción relacionada a las políticas preventivas que aplica la institución.

Los estudios encontrados sobre las variables gestión administrativa y satisfacción laboral tienen origen en los países de América Latina, como México, Perú, Chile y Ecuador, siendo México y Perú los que tienen mayores investigaciones sobre las mismas; haciendo ver que en ellos existe el interés por enriquecer los procesos que ayudan a las instituciones a tener colaboradores satisfechos y motivados.

Los artículos seleccionados permiten establecer que la satisfacción laboral es determinante para que los trabajadores desempeñen adecuadamente sus funciones; asimismo, la gestión administrativa debe tener todos sus componentes, planificación, control, dirección y organización, para que se cumplan los objetivos de la entidad, de la mano con el factor humano. Así como refiere, Camacho (2014), que la gestión administrativa es uno de los factores de suma importancia para manejar una institución, ya que ayuda al éxito del trabajo, empleando las cuatro funciones de la administración, empleadas por los directores para a determinar y lograr objetivos manifestados mediante el uso de seres humanos y otros recursos. Asimismo, la gestión administrativa depende mucho de la forma en como los jefes y gerentes, ejercen su función, en cómo desarrollan sus habilidades de liderazgo, más que por el tema de gestión administrativa en su conjunto, lo cual hace que se tenga trabajadores satisfechos, según lo manifestado por Mendoza (2017), es un proceso ordenado, dinámico, coherente y participativo; tiene acciones integrales que buscan el logro de los objetivos a través del cumplimiento de las funciones de gestión en el proceso administrativo, que hace que se tomen adecuadas decisiones dentro de las organizaciones.

Por otro lado, en la mayoría de las investigaciones analizadas, los trabajadores se sienten satisfechos a nivel medio, con sus trabajos, debido a que existen adecuadas condiciones físicas, adecuados horarios y desarrollo personal dentro de la organización. Sin embargo, existe una similitud en cuanto a la insatisfacción laboral, respecto a las condiciones físicas para ejercer la función laboral, gestión del presupuesto, salario, falta de comunicación y capacitación. Lo que genera falta de motivación, haciendo que las personas no ejerzan con entusiasmo sus funciones diarias. El hecho de que una persona este satisfecha laboralmente, se refleja en sus áreas de trabajo, pues muestran motivados y a gusto en su ambiente de 
trabajo, lo cual se refleja en la calidad del servicio y en las adecuadas relaciones interpersonales, que experimenta día a día el trabajador, un trabajador satisfecho contribuye adecuadamente al cumplimiento de los objetivos de la institución (Face y Herrera, 2017; Olivarez, et.al, 2016; Chiang y San Martín, 2015).

Por otro lado, podemos decir que la adecuada gestión administrativa permite que las personas se desarrollen personal y profesionalmente, con ello ayudan al cumplimiento de los objetivos institucionales, en vista de que se sienten reconocidos y cuidados. En la actualidad se habla y valora mucho el tema de los recursos humanos, la modernización de la gestión pública, enfocada en las competencias, hace ver que el recurso humano es el corazón de las instituciones; muchas empresas ya están encaminadas hacia esa adecuada gestión. Desde hace algunos años se aplica la evaluación de desempeño, el cual tuvo buenos resultados en muchas instituciones, porque permitió identificar talentos y mejorar la gestión de los recursos humanos; a pesar de que en un inicio los colaboradores lo vieron como algo negativo. Ahí cumplió un papel importante la sensibilización y difusión de los responsables de estos procesos.

\section{CONCLUSIÓN O CONSIDERACIONES FINALES}

La satisfacción laboral en la gestión administrativa es como se siente el trabajador en su ambiente de trabajo, los trabajadores de estas instituciones no están del todo satisfechos, por las bajas remuneraciones, los ambientes de trabajo inadecuados y el deficiente liderazgo.

La gestión administrativa necesita de estrategias que permita direccionar la organización, pues eso ayuda al cumplimiento de metas, la dirección, organización, control y planificación deben ser utilizados adecuadamente.

La satisfacción laboral es direccionada por el área de los recursos humanos quien toma en cuenta a los colaboradores como el centro de toda organización para reconocer y motivar el desempeño laboral.

\section{LISTA DE REFERENCIAS}

Bendezú, K. (2020). Gestión del talento humano y la satisfacción laboral de los trabajadores de una institución educativa privada. (Artículo científico). Perú. Revista de Investigación Valdizana, vol. 14 núm. (1): 22-28. Recuperado de: http://www.redalyc.org/articulo.oa?id=586062237004 
Caján, J. (2017). Modelo de gestión pedagógica basado en el liderazgo transformacional para garantizar la satisfacción laboral de los docentes del ISEP "Sagrado Corazón de Jesús”. (Artículo científico). Perú. UCV-HACER Revista de investigación y cultura, vol. 5 núm. (2). Recuperado de: https://www.redalyc.org/articulo.oa?id=521754663010

Chiang, M. y San Martín, N. (2015). Análisis de la satisfacción y el desempeño laboral en los funcionarios de la Municipalidad de Talcahuano. (Artículo científico). Chile. Revista Ciencia y trabajo, vol. 17 núm. (54): 159-165. Recuperado de: https://scielo.conicyt.cl/pdf/cyt/v17n54/art01.pdf

Córdova, J. y Arévalo, L. (2018). Gestión administrativa y su relación en la satisfacción laboral de los trabajadores de la distribuidora Tulipan S.A.C, ciudad de Tarapoto, año 2017. (Tesis de posgrado). Universidad Nacional de San Martín, TarapotoPerú. Recuperado de: http://repositorio.unsm.edu.pe/bitstream/handle/11458/3015/ADMINISTRACION $\% 20-$

\%20J\%C3\%A1nice\%20Ivone\%20C\%C3\%B3rdova\%20Flores\%20\%26\%20Lessli e $\% 20$ Miriella\%20Ar\%C3\%A9valo\%20Portilla.pdf?sequence=1\&isAllowed=y

Face, M. y Herrera, S. (2017). La gestión administrativa y su incidencia en la satisfacción laboral. (Artículo científico). México. Revista electrónica sobre tecnología, educación y sociedad, vol. 4 núm. (7). Recuperado de: https://pdfs.semanticscholar.org/27a2/74f08a7ce445c96ab2c16c9c2bbe0f2f2639.p df

Hernández, Fernández y Baptista (2014). Metodología de la Investigación. Sexta edición, México. Recuperado de: http://observatorio.epacartagena.gov.co/wpcontent/uploads/2017/08/metodologia-de-la-investigación-sextaedicion.compressed.pdf

Montes de Oca, J. y Pulla, C. (2019). La gestión administrativa y su incidencia en la productividad de las microempresas ecuatorianas. Caso de estudio taller Dipromax de la ciudad de Santo Domingo. (Artículo científico). República Dominicana. Revista Dilemas Contemporáneos: Educación, Política y Valores, vol. 7núm.(1):1-14.Recuperadode: 
http://www.dilemascontemporaneoseducacionpoliticayvalores.com/index.php/dile mas/article/view/1191/126

Montoya, P., Bello, N., Bermúdez, N., Burgos, F., Fuentealba, M. y Padilla, A. (2017). Satisfacción Laboral y su Relación con el Clima Organizacional en Funcionarios de una Universidad Estatal Chilena. (Artículo científico). Chile. Revista Ciencia y trabajo, vol. 19 núm. (58): 7-13. Recuperado de: https://scielo.conicyt.cl/pdf/cyt/v19n58/0718-2449-cyt-19-58-00007.pdf

Moreno, B. et al. (2018). Revisiones Sistemáticas: definiciones y nociones básicas. (Artículo científico). Revista Clínica Periodoncia Implantol, vol. 11 núm. (3): 184186. Recuperado de: https://scielo.conicyt.cl/pdf/piro/v11n3/0719-0107-piro-1103-184.pdf

Omar, A., Salessi, S. y Urteaga, F. (2017). Impact of management practices on job satisfaction. (Artículo científico). Brasil. Revista de Administração Mackenzie, vol. 18, núm. (5): 92-115. Recuperado de: http://www.redalyc.org/articulo.oa?id=195453538005

Olivares, J. et al. (2016). Satisfacción laboral de docentes universitarios del departamento académico de clínica estomatología. (Artículo científico). Perú. Revista de Estomatología Herediana, vol. 16 núm. (1): 21-25. Recuperado de: http://www.redalyc.org/articulo.oa?id=421539345005

Pacheco, R. Robles, C. y Ospino, A. (2018). Análisis de la Gestión Administrativa en las Instituciones Educativas de los Niveles de Básica y Media en las Zonas Rurales de Santa Marta, Colombia. (Artículo científico). Colombia. Revista información tecnológica, vol. 29 núm. (5): 259-266. Recuperado de: https://scielo.conicyt.cl/scielo.php?script=sci_abstract\&pid=S071807642018000500259\&lng=en\&nrm=iso\&tlng=es

Ramírez, A. y Benítez, J. (2016). Satisfacción laboral en una institución educativa de nivel superior del Estado de México. (Artículo científico). México. Revista acta universitaria, vol. 22 núm. (8): 33-42. Recuperado de: http://www.redalyc.org/articulo.oa?id=41625070002

Rodríguez, A., Gómez, P. y De Dios, R. (2017). Estudio de la satisfacción laboral en los equipos de atención primaria en un área sanitaria de Asturias. (Artículo científico). España. Revista electrónica trimestral de enfermería: Enfermería 
Global, vol. 16 núm. (3): 369-383. Recuperado de: http://dx.doi.org/10.6018/eglobal.16.3.256641

Sánchez, S. Fuentes, F. y Artacho, C. (2018). Rankings internacionales de satisfacción laboral. Capital Humano, núm. (219):60-61. Universidad de Córdova. Recuperado de:

https://factorhuma.org/attachments_secure/article/1888/c212_060_a_Rankings\%2 Ointernacionales_219_2.pdf

Sánchez, M. y García, M. (2017). Satisfacción laboral en los entornos de trabajo. Una exploración cualitativa para su estudio. (Artículo científico). Perú. Revista Scientia Et Technica, vol. 22 núm. (2):161-166. Recuperado de: http://www.redalyc.org/articulo.oa?id=84953103007

Torres, O., Vallejos, A. y Burbano, J. (2019). Buenas prácticas de gestión administrativa en empresas de servicios en la ciudad de Ibarra. (Artículo científico). Ecuador. Revista Dilemas Contemporáneos: Educación, Política y Valores, vol. 7 (1). Recuperado de: http://www.dilemascontemporaneoseducacionpoliticayvalores.com/index.php/dile mas/article/view/1043/122

Ulloa, E. y Oliver, E. (2013). Plan de gestión administrativa-financiera eficiente y eficaz para generar la Universidad Cesar Vallejo, Trujillo 2013. (Artículo científico). Perú. Revista UCV-Scientia, vol. 5 núm. (2): 214-221. Recuperado de: http://revistas.ucv.edu.pe/index.php/UCV-SCIENTIA/article/view/246 